\title{
Myocardial Remodeling in Ischemic Heart Disease - Imaging from Cell to Organ Level by Synchrotron Propagation Based X-Ray Phase-Contrast Imaging
}

Ivo Planinc ( $\square$ ivo.planinc@gmail.com )

University of Zagreb School of Medicine, University Hospital Centre Zagreb, Department of

Cardiovascular Diseases

Patricia Garcia-Canadilla

Institut d'Investigacions Biomèdiques August Pi i Sunyer (IDIBAPS), Barcelona

Hector Dejea

Institute for Biomedical Engineering

Ivana llic

University of Zagreb School of Medicine, University Hospital Centre Zagreb, Department of Pathology,

Zagreb

\section{Eduard Guasch}

Arrhythmia Unit, Department of Cardiology, Hospital Clínic de Barcelona, Barcelona

\section{Monica Zamora}

BCNatal, Hospital Clínic and Hospital Sant Joan de Déu, Barcelona,

\section{Fatima Crispi}

BCNatal, Hospital Clínic and Hospital Sant Joan de Déu, Barcelona,

\section{Marco Stampanoni}

Institute for Biomedical Engineering

\section{Davor Milicic}

University of Zagreb School of Medicine, University Hospital Centre Zagreb, Department of

Cardiovascular Diseases

\section{Bart Bijnens}

Institució Catalana de Recerca i Estudis Avançats

\section{Anne Bonnin}

Paul Scherrer Institute

\section{Maja Cikes}

University of Zagreb School of Medicine, University Hospital Centre Zagreb, Department of Cardiovascular Diseases

\section{Research Article}


Keywords: infarct remodeling, ischemic heart disease, imaging, X-ray, myocardial structure.

Posted Date: February 4th, 2021

DOI: https://doi.org/10.21203/rs.3.rs-156227/v1

License: (c) (1) This work is licensed under a Creative Commons Attribution 4.0 International License. Read Full License

Version of Record: A version of this preprint was published at Scientific Reports on July 7th, 2021. See the published version at https://doi.org/10.1038/s41598-021-93054-6. 


\section{Abstract}

Background: Cardiovascular research is in an ongoing quest for a superior imaging method to integrate gross-anatomical information with microanatomy, combined with quantifiable parameters of cardiac structure. In recent years, synchrotron radiation-based X-ray Phase Contrast Imaging (X-PCI) has been extensively used to characterize soft tissue in detail.

The objective was to use X-PCI to comprehensively quantify ischemic remodeling of different myocardial structures, from cell to organ level, in a rat model of myocardial infarction.

Methods and Results: Myocardial infarction-induced remodeling was recreated in a well-established rodent model. Ex vivo rodent hearts were imaged by propagation based X-PCl using two configurations resulting in $5.8 \mu \mathrm{m}$ and $0.65 \mu \mathrm{m}$ effective pixel size images. The acquired datasets were used for a comprehensive assessment of macrostructural changes including the whole heart and vascular tree morphology, and quantification of left ventricular myocardial thickness, mass, volume, and organization. On the meso-scale, tissue characteristics were explored and compared with histopathological methods, while microstructural changes were quantified by segmentation of cardiomyocytes and calculation of cross-sectional areas.

Conclusions: Propagation based X-PCI provides detailed visualization and quantification of morphological changes on whole organ, tissue, vascular as well as individual cellular level of the ex vivo heart, with a single, non-destructive 3D imaging modality.

\section{Introduction}

While ischemic heart disease is one of the most studied cardiac pathologies, many questions remain regarding the understanding of myocardial remodeling with improved treatment and more tissue salvage as the ultimate goal. To develop protective or tissue repair strategies, the in-depth understanding of the underlying pathophysiological processes and the involved structural changes of the heart are of paramount importance. Ideally this is amalgamated in an imaging modality enabling the integration of structural information from cell to organ level.

Cardiac remodeling is a combined response of cardiomyocytes and other cells, myocardial tissue, and the heart as an organ, to different adverse pathophysiological processes (such as loss of contractile force by necrosis or apoptosis of cardiomyocytes, cellular hypertrophy, intercellular matrix changes like fibrosis, or deposition of different metabolic end-products). ${ }^{1}$ These processes may result in changes in cardiac size, myocardial mass, (segmental) geometry, cardiomyocytes appearance and orientation, and ultimately function of the whole organ, thus having a profound impact on patient outcome. One of the typical examples of negative cardiac remodeling is induced by ischemic heart disease, with a myocardial infarction (MI) being the trigger of an adverse cascade. Major insights in the remodeling processes have been gained from animal models of cardiovascular disease (either rodents, or large animals such as 
sheep and swine) where using ex vivo gross anatomical morphology or histology have improved our knowledge of the induced changes. $2,3,4,5$

In clinical practice, ischemic heart disease is assessed by means of diagnostic imaging modalities, encompassing those that visualize the coronary arteries, but also that quantify the resulting adverse remodeling: coronary angiography, echocardiography, magnetic resonance imaging, computed tomography, and nuclear cardiology imaging are often combined as complementary techniques. Histopathological analysis is seldom utilized in routine clinical assessment of myocardial remodeling, but remains a standard research tool for detailing tissue and cell structure, while additional methods of histochemical staining help differentiate the expression of specific cellular or extracellular signals in tissues. Cardiovascular research, as well as clinical cardiology, are in an ongoing quest for a superior (imaging) method that could integrate gross-anatomical information with microanatomy, combined with quantifiable parameters of cardiac structure.

In recent years, synchrotron radiation-based X-ray Phase Contrast Imaging (X-PCI) has been extensively used to characterize soft tissue in detail. Synchrotrons are large scale facilities that generate highly collimated and brilliant X-ray beams. By exploiting the refractive properties of these beams in dedicated setups, $\mathrm{X}-\mathrm{PCl}$ is able to image soft tissues or organs non-destructively in 3D and at high-resolution (down to $<1 \mu \mathrm{m}$ ), with increased contrast in comparison to conventional absorption imaging. Particularly in the cardiovascular field, X-PCl has so far been utilized for ex vivo imaging of animal models, human fetus hearts and transmural samples of adult human hearts, both normal and diseased. $6,7,8,9,10,11,12,13,14,15,16$

The aim of our study was to use X-PCl to comprehensively quantify remodeling of different myocardial structures, from cell to organ level, as a consequence of $\mathrm{MI}$ in a rat model.

\section{Results}

\section{Macro scale}

\section{Whole heart morphology - quantification of LV myocardial thickness, mass and volume}

The obtained 3D datasets can be easily used to assess the gross anatomical features of imaged hearts. In Fig. 1, the low resolution (LR) propagation-based (PB) X-PCl datasets were re-sliced to obtain the conventional imaging views, allowing clear visualization of the cardiac chambers, identification of the segments of the left ventricle (LV) and right ventricle (RV), as well as the different valve leaflets and details of the trabeculations. The whole heart acquisitions allow to clearly appreciate the exact extension of myocardial scar: in the right panel of Fig. 1, the infarct extends through most of the anterior, anterolateral and inferolateral LV walls, indicating a very large perfusion area of the ligated left coronary artery. 
Supplemental online Figure I shows an example of the 45-segment model of one of the infarcted hearts indicating the normal (contralateral), peri-, and $\mathrm{Ml}$ area.

When quantifying LV cavity volumes, myocardial mass and wall thickness; thinner walls, larger cavity volumes and increased myocardial mass were observed in the infarcted hearts as compared to the control rat heart (Table 1 and Fig. 2).

Table 1

Parameters and quantification of global and cellular LV remodeling obtained by PB X-PCl imaging.

Myocardial (global) remodeling

\begin{tabular}{|c|c|c|c|c|c|c|}
\hline & $\begin{array}{l}\text { Control rat } \\
\text { heart }\end{array}$ & $\begin{array}{l}\text { Post MI } \\
\text { Average }\end{array}$ & $\begin{array}{l}\text { Ml } \\
\text { Heart } \\
1\end{array}$ & $\begin{array}{l}\text { MI } \\
\text { Heart } 2\end{array}$ & $\begin{array}{l}\text { MI } \\
\text { Heart } \\
3\end{array}$ & $\begin{array}{l}\text { Ml } \\
\text { Heart } \\
4\end{array}$ \\
\hline Wall thickness (mm) & $2.2 \pm 0.4$ & $1.8 \pm 0.8^{*}$ & $\begin{array}{l}1.9 \pm \\
0.9\end{array}$ & $\begin{array}{l}2.1 \pm \\
0.9\end{array}$ & $\begin{array}{l}1.8 \pm \\
0.7\end{array}$ & $\begin{array}{l}1.5 \pm \\
0.7\end{array}$ \\
\hline LV cavity volume (ml) & 0.11 & $\begin{array}{l}0.26 \pm \\
0.09 *\end{array}$ & 0.20 & 0.20 & 0.23 & 0.39 \\
\hline LV myocardial volume (ml) & 0.58 & $\begin{array}{l}0.62 \pm \\
0.02^{\star}\end{array}$ & 0.62 & 0.63 & 0.60 & 0.63 \\
\hline LV myocardial mass (g) & 0.61 & $\begin{array}{l}0.67 \pm \\
0.03^{*}\end{array}$ & 0.71 & 0.67 & 0.63 & 0.66 \\
\hline \multicolumn{7}{|l|}{ Cellular remodeling } \\
\hline \multicolumn{7}{|l|}{$\begin{array}{l}\text { Cardiomyocyte cross } \\
\text { sectional area }\left(\mu \mathrm{m}^{2}\right)\end{array}$} \\
\hline Septum & $312 \pm 62+$ & $499 \pm 141 \dagger$ & $\begin{array}{l}477 \pm \\
116^{\star}\end{array}$ & $\begin{array}{l}521 \pm \\
160^{\star}\end{array}$ & - & - \\
\hline Anterior wall & $330 \pm 92 \dagger$ & $737 \pm 127 \dagger$ & $\begin{array}{l}773 \pm \\
118^{\star}\end{array}$ & $\begin{array}{l}701 \pm \\
126^{\star}\end{array}$ & - & - \\
\hline Overall & $321 \pm 75+$ & $618 \pm 179+$ & $\begin{array}{l}625 \pm \\
194\end{array}$ & $\begin{array}{l}611 \pm \\
168\end{array}$ & - & - \\
\hline
\end{tabular}

Upper panel: LV wall thickness is averaged over 45 segments for each animal. LV- left ventricle, MImyocardial infarction. *denotes statistical significance $(p<0.05)$ for comparison of means between post-MI hearts and the healthy heart.

Lower panel: * significant difference in CSA between different myocardial areas in the same heart ( $p$ $\leq 0.01$ ). tsignificant difference in CSA overall and between similar myocardial areas of affected and non-affected hearts $(\mathrm{p} \leq 0.01)$. Ml- myocardial infarction.

$p$ values in this table were obtained using Student's T-test.

\section{Quantification of myocardial organization}


For further insight into the structural changes occurring as a consequence of $\mathrm{MI}$, we have quantified local cardiomyocyte aggregates orientation. A longitudinal view of the local helical angles $(\mathrm{HA})$ values of an infarcted and control rat heart is shown in Fig. 3.

As expected, the HAs in control heart show predominance of positive angles in the endocardium with a smooth progression to negative angles when approaching the epicardium. A similar pattern of cardiomyocyte organization can be seen in the non-affected segments of the post-MI heart. In the infarct zone, the HA still show the 'normal' positive values at the endocardium (purple/dark blue color in Fig. 3), and negative values (green/yellow) at the epicardium, even in the segments of clear transmural scar. Preservation of the general cardiomyocyte orientation indicates certain amount of preserved cardiomyocyte aggregates in the endocardial and epicardial areas of the infarct zone. The results of linear fitting of the transmural HA for LV segments are shown in supplementary online Table I.

Furthermore, the analysis of myocardial disarray index (MDI) shows clear differences in the local cardiomyocytes organization between different regions of the post-MI heart, as well as between the control rat heart and post-MI hearts in general (Table 2.). MI segments have the lowest values of MDI indicating high local myocardial disarray. Also, all segments of the post-MI rat hearts have lower MDI values (i.e. more disarray) in comparison to the control rat heart (precisely, significantly lower for MI, peri$\mathrm{MI}$, and remaining segments, and borderline significant for contralateral segments). 
Table 2

Myocardial disarray index (MDI) in different regions of all hearts. Significant differences are observed in

$\mathrm{MDI}$ between post-MI and control, as well as between different regions of post-MI hearts.

Myocardial disarray index (MDI)

\begin{tabular}{|c|c|c|c|c|c|c|}
\hline & Control & Post-MI & $\begin{array}{l}\text { Ml Heart } \\
1\end{array}$ & $\begin{array}{l}\text { Ml Heart } \\
2\end{array}$ & $\begin{array}{l}\text { MI Heart } \\
3\end{array}$ & $\begin{array}{l}\text { Ml Heart } \\
4\end{array}$ \\
\hline Ml segments & - & $\begin{array}{l}0.76 \pm \\
0.06^{*}\end{array}$ & $\begin{array}{l}0.74 \pm \\
0.06\end{array}$ & $\begin{array}{l}0.82 \pm \\
0.05\end{array}$ & $\begin{array}{l}0.75 \pm \\
0.06\end{array}$ & $\begin{array}{l}0.77 \pm \\
0.06\end{array}$ \\
\hline Peri-MI segments & - & $\begin{array}{l}0.87 \pm \\
0.04^{\star *}\end{array}$ & $\begin{array}{l}0.87 \pm \\
0.03\end{array}$ & $\begin{array}{l}0.87 \pm \\
0.04\end{array}$ & $\begin{array}{l}0.83 \pm \\
0.07\end{array}$ & $\begin{array}{l}0.88 \pm \\
0.03\end{array}$ \\
\hline $\begin{array}{l}\text { Contralateral } \\
\text { segments }\end{array}$ & - & $\begin{array}{l}0.88 \pm \\
0.04^{\star \neq} \neq\end{array}$ & $\begin{array}{l}0.88 \pm \\
0.03\end{array}$ & $\begin{array}{l}0.87 \pm \\
0.02\end{array}$ & $\begin{array}{l}0.86 \pm \\
0.08\end{array}$ & $\begin{array}{l}0.90 \pm \\
0.03\end{array}$ \\
\hline $\begin{array}{l}\text { Remaining } \\
\text { segments }\end{array}$ & - & $\begin{array}{l}0.87 \pm \\
0.04^{\star \star}+\end{array}$ & $\begin{array}{l}0.85 \pm \\
0.04\end{array}$ & $\begin{array}{l}0.94 \pm \\
0.04\end{array}$ & $\begin{array}{l}0.86 \pm \\
0.05\end{array}$ & $\begin{array}{l}0.86 \pm \\
0.05\end{array}$ \\
\hline Overall & $\begin{array}{l}0.89 \pm \\
0.04^{\star}\end{array}$ & $\begin{array}{l}0.83 \pm \\
0.07 \dagger\end{array}$ & $\begin{array}{l}0.82 \pm \\
0.13\end{array}$ & $\begin{array}{l}0.86 \pm \\
0.05\end{array}$ & $\begin{array}{l}0.82 \pm \\
0.08\end{array}$ & $\begin{array}{l}0.83 \pm \\
0.07\end{array}$ \\
\hline \multicolumn{7}{|c|}{$\begin{array}{l}\text { *p }<0.01 \text { for comparison between MDI in MI segments and peri-MI, contralateral, and remaining } \\
\text { segments, as well as in comparison with overall MDI in control. }\end{array}$} \\
\hline \multicolumn{7}{|c|}{$\begin{array}{l}\mathrm{tp}<0.01 \text { for comparison between MDI in the control heart and peri-MI, and remaining segments, as } \\
\text { well as in comparison with overall MDI in post-Ml hearts. }\end{array}$} \\
\hline \multicolumn{7}{|c|}{$\neq \mathrm{f}=0.05$ for comparison between MDI in control heart and contralateral segments of post-MI hearts. } \\
\hline \multicolumn{7}{|c|}{$\begin{array}{l}\text { All values are expressed as mean } \pm \text { standard deviation. } p \text { values in this table were obtained using } \\
\text { Student's T-test. }\end{array}$} \\
\hline
\end{tabular}

\section{Vascular tree}

The segmentation and subsequent visualization of the vascular tree allowed to identify the precise location of left coronary artery occlusion. It also showed a lack of collateral circulation in the $\mathrm{Ml}$ area, confirming the development of the transmural MI. On the other hand, it also showed increased vascular spaces in the infarct area that may represent various stages of angiogenesis and a basis for future (retrograde) collateral circulation formation (Fig. 4). Similar to some previous reports, the vascular spaces in the infarcted area seem larger, and with less defined vascular walls possibly indicating a venous origin or extensive branching processes of capillaries (Supplemental online videos I, II, and III). ${ }^{17,18,19}$

\section{Mesoscale}

\section{Local tissue characterization}

Several specific myocardial segments were used for high resolution (HR) imaging, serving as a form of virtual histology, and compared to classical assessment. At the effective pixel size of $0.65 \mu \mathrm{m}$, and in the whole heart, with no further specimen preparation or staining, the cardiomyocytes, forming the local 3D 
myocardial micro-organization, could clearly be visualized. The intercellular matrix, containing fibrous tissue and vasculature, was also easily identified. Figure 5 shows the comparison of the same myocardial area imaged by virtual histology PB X-PCl, and conventional histology. Besides close resemblance of the images produced by these two different techniques, the information content is similar. We may clearly observe the preserved longitudinally arranged cardiomyocytes on the endocardial side, and circumferentially arranged towards the mesocardium. Virtual histology obtained by PB X-PCl, due to its three-dimensionality, allows for easy tracking of entire cardiomyocytes bundles, providing information on its interrelation with the overall myocardial structure.

To illustrate the potential for tissue characterization, Fig. 6 compares the whole short-axis slice LR PB X$\mathrm{PCl}$ versus different histology staining of the control and an infarcted heart.

Figure 5 (right panel) shows this in more detail comparing the LR and HR PB X-PCl image with histology at larger magnification.

In the early phases of an MI, reticular fibers (non-mature forms, rich in collagen type III) are usually present, representing the ongoing healing process. In our samples, reticular fibers could not be visualized, confirming that the scarring process of the infarcted areas has been finalized, and that true transmural scars are formed (Fig. 5, 6).

\section{Microscale}

\section{Quantification of cellular remodeling}

The HR level achieved by PB X-PCI allowed identification and quantification of individual cardiomyocyte size. While very few cardiomyocytes were present in the Ml zones (and were thus not quantified), in all other regions cardiomyocyte hypertrophy was present, with larger average cross-sectional area (CSA) in the post-MI hearts in contrast to control rat heart $\left(737 \pm 127 \mu \mathrm{m}^{2}\right.$ (anterior wall) and $499 \pm 141 \mu \mathrm{m}^{2}$ (septum) vs. $330 \pm 92 \mu \mathrm{m}^{2}$ and $\left.312 \pm 62 \mu \mathrm{m}^{2}\right)$, indicating a significant $(\mathrm{p}<0.001)$ difference between peri$\mathrm{MI}$ and contralateral areas of post-MI hearts, as well as compared to the control rat heart (Table 1). Cardiomyocyte CSA were consistent between post-MI hearts 1 and 2 (anterior wall: $773 \pm 118 \mu \mathrm{m}^{2}$ (postMl rat 1 ) vs. $701 \pm 125 \mu \mathrm{m}^{2}$ (post-MI rat 2), $\mathrm{p}=0.07$ and septum: $477 \pm 116 \mu \mathrm{m}^{2}$ (post-MI rat 1) vs. $521 \pm$ $160 \mu \mathrm{m}^{2}$ (post-MI rat 2), $\mathrm{p}=0.255$ ).

\section{Discussion}

In this manuscript, we present a comprehensive assessment of the macro-, meso-, and micro-structural changes caused by $\mathrm{MI}$ in an ex vivo rat model of left coronary artery ligation. PB X-PCl provides visualization and quantification of morphological changes on whole organ, tissue organization and type, vascular as well as individual cellular level, with a single, non-destructive 3D imaging modality, in the absence of further specimen manipulation, contrast agents or tissue staining. 
The entirety of information gained by PB X-PCl in an animal model of ischemic heart disease would conventionally require using several different imaging modalities in conjunction with (destructive) histopathological analysis. Whole-heart morphology and quantification of cardiac remodeling is usually obtained by means of echocardiography, (micro-) computed tomography, or magnetic resonance imaging in in vivo research animal models or in clinical medicine. ${ }^{2,20}$ For the analysis of changes at the cellular level, light and electron microscopy are the routine methods. While confocal or transmission microscopy offers some 3D characterization at the cellular level, imaging depth is still a major limitation and tissue preparation remains cumbersome. ${ }^{21}$ Automated (ion-beam or others) slicing methods help, but only up to a certain point. ${ }^{22,23}$ The major drawbacks of histological procedures therefore include time-consuming sample preparation, usually demanding destruction of tissue, as well as different staining methods. Additionally, the need for thin slices makes 3D assessment of the analyzed structure very difficult since the off-line reconstructions of 3D structures are often performed from 2D images, with problems with slice loss or distortion. ${ }^{21}$

In recent years, X-ray computed tomography using synchrotron radiation has emerged and has been validated for imaging of different cardiac pathologies by assessing native intact animal heart models, as well as ex vivo human fetal hearts, and transmural samples of adult human hearts, both normal and disease-affected (such as fetuses with congenital heart disease). $6,7,8,9,10,11,12,13,14,15,16$ For example, Gonzalez-Tendero et al. illustrated that PB X-PCI can quantify cardiac structures and tissue with enough details to reveal microfiber and vascular structure throughout whole rodent and rabbit fetal hearts, focusing on remodeling associated with intrauterine growth restriction. ${ }^{6,15}$ They showed that the great advantage of PB X-PCl, as compared to micro-computed tomography (micro-CT), is that it provides a better contrast between soft cardiac tissues than classical X-ray absorption imaging.

In this study, we have shown that PB X-PCl allows for multiscale quantification of the precise regional and transmural extension of an $\mathrm{Ml}$, from organ to cellular level. At the macro scale, it offers the quantification of global cardiac remodeling, such as wall thickness, myocardial mass and cavity volumes, as well as the visualization of the coronary arterial and venous tree, enabling the precise identification of the location of the left coronary artery occlusion. While global remodeling in the setting of an $\mathrm{Ml}$ is well understood, the whole heart macro-vascular assessment offered by PB X-PCI allows new avenues to explore. For example, the adaptive response and regeneration of the vasculature can be clearly seen from the increased vascular branching and the presence of enlarged thin wall vessels in the area of the MI. These large vessels were described before using histology and their overall effect studied by MRI, but PB X-PCl allows the direct visualization and quantification in the whole 3D infarct area where both the arterial as well as the venous branches can be studied without the need for contrast agents. ${ }^{24} \mathrm{At}$ $\mathrm{HR}$, even some properties of the vascular walls can be appreciated.

At the meso-scale, specific magnetic resonance techniques, such as diffusion tensor imaging (DT-MRI) and tractography, have been used to characterize in vivo and ex vivo the transmural course of the orientation of cardiomyocyte aggregates ("fibers") in 3D - an important component of cardiac architecture 
that might be affected by different cardiac conditions, such as MI. However, compared to the size of cardiomyocyte, DT-MRI has a limited resolution (with the smallest achieved pixel sizes many times larger than individual cardiomyocytes) and specifically assesses diffusivity in water-containing structures, which might not make it the ideal modality to investigate scarred or highly fibrotic tissue or when assessing intracellular matrix remodeling. Additionally, the visual representations of fiber-tracks obtained by tractography do not directly show all local properties and are affected by imaging artefacts. ${ }^{25,26}$

As opposed to DT-MRI, PB X-PCl, with a much better resolution and assessment of the non-watery structure, enables direct visualization, as well as local quantification, of cardiomyocyte aggregates and their orientation, thus providing insight to the local organization of the myocardium in detail.

From the same whole heart imaging setup, combined with our multiscale approach, PB X-PCl also enabled to study adaptations at the cellular level and assess cellular remodeling by quantifying hypertrophy of individual cardiomyocytes in different myocardial regions. Similarly to what was shown before from histopathological studies, we measure significant differential hypertrophy induced by the presence of an $\mathrm{Ml}$, both in the preserved myocardium adjoining the infarct, as well as in contralateral (distant) myocardial regions, however more pronounced in the former area. ${ }^{1}$ Combining the different scales (as shown in Fig. 5) provides a novel, more integrated approach towards assessing infarct remodeling, its mechanisms and the potential therapeutic benefits. There is still some controversy about the formation of an infarct and the resulting tissue structure. Studies using (DT-) MRI, suggested a clear decrease of local organization and absence of "fiber"-directions in the infarct. ${ }^{27}$ Others, using a different analysis approach, suggested that the global orientation of myocardial sheets is preserved. ${ }^{28}$ These discrepancies likely originate from the principle of MRI, predominantly assessing the presence and dynamics of water molecules in cardiomyocytes and the intercellular space. ${ }^{29}$

Using classical histopathology approaches, it was shown that despite a large increase in collagen density, the organization is maintained in the core of infarction in both acute and chronic Ml. While collagen is clearly synthesized in the infarction, its orientation (just like the initial 'fiber' directions during cardiac development) is influenced by mechanical loading direction. ${ }^{30,31,32,33}$

As can be seen from Fig. 3, our images as well as the quantitative regional 'fiber' analysis focused on small tissue volumes, show tissue organization in the infarct. While there is a small, but significant, difference in the MDI between regions affected by MI, peri-MI areas, and contralateral areas, as well as between $\mathrm{Ml}$-affected and control rat hearts, the overall organization is clearly preserved. Although only very few cardiomyocytes remain in the area of transmural $\mathrm{Ml}$, the intercellular meshwork is still similar in structure and show a clear preserved organization, with gradually changing direction from epi- to endocardium. This suggests that the lack of perfusion results in the disappearance of cardiomyocytes and a collapse of the matrix. Interestingly, both at the endocardial as well as epicardial border, a fully intact myocardial layer with a thickness of several cardiomyocytes remains present, likely thanks to the perfusion by diffusion from the blood in the cavity and the pericardial fluid. 
In summary, in this paper we have shown that PB X-PCl offers a powerful research tool to study tissue remodeling following an $\mathrm{MI}$ using a whole heart, 3D, non-destructive, non-staining or contrast-based technique, feasible for the quantification of myocardial remodeling at whole-organ, tissue, vascular as well as cellular level in ex vivo model of ischemic heart disease.

\section{Limitations}

Synchrotrons are large scale facilities with limited access due to high competition for the available time slots. Although this currently limits the amount of studies that can be performed, there is a clear technical development pathway towards using PB X-PCI based on laboratory X-ray sources or alternative laserelectron beam setups. Micro-CTs with X-PCI capabilities can be expected in the near future. $34,35,36,37,38,39,40$

Also, while studies on imaging beating hearts (Langendorff and in-vivo) with X-PCI are underway, the current research was performed on static, ex-vivo hearts thus lacking direct correlative function assessment, and lacking direct comparison with in vivo methods.

Limited number of animal models of disease and one control animal hinder the validation power of this study, however the aim was primarily to show the comprehensive potential of the method in the setting of ischemic heart disease pathological processes.

Finally, the current field-of-view of most synchrotron imaging beamlines only allows to study small (rodent and human fetal) specimens given that scan time importantly increases when stitched acquisitions need to be performed.

\section{Methods}

\section{Animal model}

Post-MI remodeling was recapitulated in a well-established rodent model. A transmural MI was induced by left coronary artery ligation through a left thoracotomy in 8 to 11 week-old Wistar male rats (Charles River Laboratories, France, EU). ${ }^{41}$ Rats were sacrificed after 2 weeks, the hearts were excised, and immersed in formalin. Rats were euthanized with an anaesthetic overload (5\% inhaled isoflurane) and subsequent exsanguination. For this experiment 4 rats with induced $\mathrm{MI}$, and 1 control healthy rat were used. Online Supplementary methods explain in more detail animal handling.

Animal care and experimentation conformed to the European Union (Directive 2010/63/UE) and Spanish guidelines (RD 53/2013) for experiments involving animals. This study was approved by the local animal research ethics committee “Comité de Ética de Experimentación Animal (CEEA)" (CEEA 68/5435). The study planning, design, implementation and reporting was performed in compliance with ARRIVE guidelines. 


\section{Data acquisition}

Synchrotron radiation-based X-PCl image acquisition was carried out at the TOMCAT beamline (X02DA) of the Swiss Light Source (Paul Scherrer Institute, Switzerland). The samples were placed in dedicated tubes with degassed deionized water as medium to reduce the chance of bubble formation. The samples were then placed on the stage for acquisition with a multi-scale PB X-PCI setup. ${ }^{7}$ Briefly, the sample is fully illuminated by the X-ray beam and the microscope is placed at a certain distance from the sample in order to allow for interference patterns to form, which will induce PB contrast enhancement, especially on the interface between different tissues. In our case, a monochromatic beam with an energy of $20 \mathrm{keV}$ and two configurations were used. First, a LR configuration is chosen to image the whole heart at $5.8 \mu \mathrm{m}$ effective pixel size, with a sample-detector distance of $333 \mathrm{~cm}$, using a LuAG:Ce $300 \mu \mathrm{m}$ scintillator (Crytur, Czech Republic) and a PCO.Edge 4.2 CMOS detector (PCO AG, Kelheim, Germany). The resulting dataset is used for navigation of regions of interest to be imaged with a HR setup (at $0.65 \mu \mathrm{m}$ effective pixel size, sample-detector distance of $20 \mathrm{~cm}$, with a LuAG:Ce $20 \mu \mathrm{m}$ scintillator (Crytur, Czech Republic) and a PCO.Edge 5.5 CMOS detector (PCO AG, Kelheim, Germany)), and for offline assessment of whole organ remodeling, cardiomyocytes orientation analysis, and vasculature visualization. ${ }^{7}$

When the sample is too large to fit fully in the field of view, several overlapping scans have to be acquired and stitched a posteriori. The acquired projections are reconstructed using the Gridrec algorithm both in absorption and applying the single distance phase retrieval method developed by Paganin. ${ }^{42,43}$

\section{Image analysis}

Fiji, an open-source platform for biological-image analysis, was used to analyze and visualize gross anatomical features, as well as induced histopathological remodeling by myocardial infarction (ImageJ v.1.51s, Wayne Rasband, National Institute of Health, USA). ${ }^{44,45}$

Pre-processing of the LR datasets, such as contrast and brightness enhancement, was performed to ease the process of feature detection.

Then, a semi-automatic, whole heart segmentation was performed on the LR datasets using llastik to obtain first a rough segmentation, followed by smoothing by an in-house algorithm implemented in MATLAB (R2018b). ${ }^{46}$

\section{Left ventricular myocardial and cavity volumes calculation}

In order to calculate the LV myocardial and cavity volumes, the epicardial and endocardial borders of the LV were manually delineated in some short-axis image slices of the LR datasets from the apex to the base. Then a 3D interpolation was performed to obtain the whole LV mask using a custom algorithm implemented in MATLAB from which both LV myocardial and cavity volumes were quantified. 
For the detailed regional analysis of structural myocardial changes, a 45-segment myocardial model was used. ${ }^{47}$ Every segment was assessed for myocardial damage and categorized in three categories, defined as: 1. Transmural Ml: the whole segment is scarred, 2. peri-Ml: segments immediately adjacent to the infarcted area, with no signs of scarring, 3. contralateral segments: segments directly opposite to the infarcted ones, with no signs of loss of myocardium.

For each of the segments, LV myocardial thickness was also automatically calculated by computing the Euclidean distance transform of the binary LV mask using a custom algorithm implemented in MATLAB.

\section{Cardiomyocyte segmentation and cross-sectional area calculation}

The high resolution achieved by PB X-PCl allowed identifying and analyzing individual cardiomyocytes in the HR datasets, that were segmented and their CSA was quantified using open-source software (FijiImage $\mathrm{J}$ and $3 \mathrm{D}$ Slicer). ${ }^{48,49}$

In our cardiomyocyte analysis, we included two post-MI and the control hearts. Cardiomyocytes were analyzed in sub-volumes sized $551.2 \mu \mathrm{m} \times 551.2 \mu \mathrm{m} \times 260 \mu \mathrm{m}$ (length $\mathrm{x}$ width $\mathrm{x}$ height) of the HR datasets. In post-MI hearts, cardiomyocytes in the area of infarction (including preserved cells adjoining the fibrotic post-Ml tissue), as well as in the non-affected myocardium were analyzed. The infarcted area was part of the anterior wall, while the non-affected area analyzed was located in the septum. The same corresponding areas were also quantified in the control rat heart. Two sub-volumes were selected per area, one closer to the endocardium, and the other closer to the epicardium. Individual cardiomyocytes were segmented by manual delineation. Then CSA calculation was performed in Fiji-ImageJ and expressed as the mean value with standard deviation of measurements of 10 cells per sub-volume.

\section{Cardiomyocyte aggregates orientation}

The orientation of the local cardiomyocyte aggregates (often referred to as 'fibers') was assessed on LR datasets using a custom structure tensor (ST) based algorithm implemented in MATLAB. ${ }^{8,16}$ Briefly, for each image voxel, the gradient in $\mathrm{x}, \mathrm{y}$ and $\mathrm{z}$ directions was computed using a central difference algorithm. Then, the ST was obtained as the cross product of gradient vectors. Eigen-decomposition was applied to the ST to obtain the three eigenvalues and their corresponding eigenvectors. The eigenvector with the smallest eigenvalue (tertiary eigenvector) was considered to be the one pointing in the longitudinal direction of cardiomyocytes as it corresponds to the direction with lowest image intensity variation. The HA was calculated for each voxel as the angle between the tertiary eigenvector and the local circumferential plane.

Cardiomyocyte aggregates orientation analysis and quantification was performed across the $45 \mathrm{LV}$ myocardial segments. Transmural profiles (from endo- to epicardium or right-side endocardium) of the HAs in all of the LV segments were obtained. Linear fitting of the transmural profiles of the HA was done, and the two coefficients $\left(\beta_{1}, \beta_{0}\right)$ and the $R^{2}$ (linearity) coefficient of the linear fitting $\left(y=\beta_{1}{ }^{\star} x+\beta_{0}\right)$ were calculated. 
Local myocardial disarray was quantified by the calculation of the MDI as proposed recently by GarciaCanadilla P et al. ${ }^{9}$ This index quantifies, for each image voxel, the uniformity of the cardiomyocytes' longitudinal direction (represented by the tertiary eigenvector) within a neighborhood of size of $200 \mu \mathrm{m}^{3}$. The MDI ranges between 0 and 1, with larger values indicating highly organized cardiomyocytes (insignificant disarray) whereas lower values of MDI denote a greater loss of cardiomyocytes organization (high degree of disarray).

\section{Vasculature visualization}

PB X-PCl images show enough tissue contrast to identify vessels without the need for X-ray contrast agents. On the LR datasets the ostia of the coronary arteries were identified at the level of aortic valve, and a detailed segmentation of main coronary arteries and its main branches was performed in 3DSlicer. 48,49 The resulting 3D coronary artery tree was visualized using Seg3D and used for the identification of the exact location of the left coronary artery occlusion. ${ }^{50}$ Furthermore, the vascular branching was identified and visualized in the area of MI.

\section{Histopathology}

Following the PB X-PCI imaging of the intact hearts, a 'gold-standard' histopathological analysis was performed for comparison with the PB X-PCl images, obtained at both the cellular and organ level. The hearts were cut, embedded in paraffin and sectioned with a microtome into $4 \mu \mathrm{m}$ slices that were mounted on glass slides and stained using hematoxylin and eosin dyes. Furthermore, Mallory's trichrome and Gömöri's dyes were used to better discern mature collagen (presumably rich in collagen type I) and reticular fibers. The tissue slides were imaged using light microscopy with $0.55 \mathrm{x}$ and $20 \mathrm{x}$ magnification (approximately 2.2 and $0.7 \mu \mathrm{m}$ point-to-point resolution respectively). Furthermore, images from glass slides were digitized using the microscope camera (microscope Zeiss, model AX10/Lab.A1/; camera AxiocamERe5s; acquisition program ZEN2.5) and NanoZoomer 2.0 RS (Hamamatsu Photonics, Japan).

\section{Statistical analysis}

Continuous variables were expressed as means with standard deviation. Comparison between groups was done using two-sample Student's T-test. Global myocardial remodeling parameters between post-MI hearts and the control rat heart were compared using Student's T-test. Statistical analysis was performed using STATA (Stata/IC 13.1 for Mac, Statacorp, Texas, USA).

\section{Declarations}

\section{Acknowledgments:}

We acknowledge the Paul Scherrer Institut, Villigen, Switzerland for provision of synchrotron radiation beamtime at beamline TOMCAT of the SLS. 
We acknowledge Assoc. prof. Zeljka Krsnik, MD, PhD and the Laboratory for Digitalization of Zagreb Brain Collection of the Croatian Institute for Brain Research for support in digitalization of histology slides.

\section{Author contributions:}

I.P., P.G.C., H.D., M.S., B.B., A.B., D.M., and M.C. designed the study and planned the experiments; I.P., H.D., P.G.C., A.B. performed the imaging experiments, reconstructed the images; I.P. performed statistical analysis; H.D. performed vasculature analysis; P.G.C. analysed myocardial organization; I.I. performed histological analysis and interpretation; E.G., M.Z., F.C., prepared the animal models, handled the animals and prepared the tissues for analysis; All authors critically revised the manuscript and gave their final approval of the content.

\section{Sources of funding:}

This project was supported by the grant \#2017-303 of the Strategic Focal Area "Personalized Health and Related Technologies (PHRT)" of the ETH Domain.

\section{Disclosures:}

I.P. reports grants, personal fees and non-financial support from consulting honoraria, speaker fees and educational grants from Novartis, Pfizer, Boehringer-Ingelheim, Teva Pharmaceutical Industries, Bayer, Krka, Servier, Sandoz, Corvia and Abbott, outside the submitted work.

D.M. reports reports grants, personal fees and non-financial support from consulting honoraria, speaker fees and educational grants from Abbott, Novartis, Roche diagnostics, Bayer, Pfizer, Boehringer Ingelheim, Amgen, Sanofi, Krka, Novo Nordisk and Berlin Chemie Menarini.

M.C. reports grants, personal fees and non-financial support from Novartis, Abbott, GE Healthcare, Corvia, Pfizer, Bayer, Boehringer-Ingelheim, AstraZeneca, Sanofi, Teva Pharmaceutical Industries, Orion Pharma and Medtronic, outside the submitted work

P.G.C., H.D., I.I., E.G., M.Z., F.C., M.S., B.B. and A.B. report no potential conflict of interest.

\section{References}

1. Pfeffer, M.A., Braunwald, E.B. Ventricular remodelling after myocardial infarction. Circulation. 81, 1161-72 (1990).

2. Cohn, J.N., Ferrari, R., Sharpe, N. Cardiac remodelling-concepts and clinical implications: a consensus paper from an international forum on cardiac remodelling. On behalf of an International Forum on Cardiac Remodelling. J Am Coll Cardiol. 35, 569-82 (2000).

3. Heusch, G. et al. Cardiovascular remodelling in coronary artery disease and heart failure. Lancet. 383, 1933-43 (2014). 
4. Xie, M., Burchfield, J.S., Hill, J.A. Pathological Ventricular Remodelling: Mechanisms: Part 1 of 2. Circulation. 128, 388-400 (2013).

5. Watson, S.R., Dormer, J.D., Fei, B. Imaging Technologies for cardiac fiber and heart failure: a review. Heart Fail Rev. 23, 273-89 (2018).

6. Gonzalez-Tendero, A. et al. Whole heart detailed and quantitative anatomy, myofibre structure and vasculature from X-ray phase-contrast synchrotron radiation-based micro computed tomography. Eur Heart J-Cardiovasc Imag. 18, 732-41 (2017).

7. Dejea, H. et al. Comprehensive analysis of animal models of cardiovascular disease using multiscale X-ray phase contrast tomography. Sci Rep. 9, 6996 (2019).

8. Garcia-Canadilla, P. et al. Complex congenital heart disease associated with disordered myocardial architecture in a midtrimester human fetus. Circ Cardiovasc Imaging. 11, e007753;

10.1161/CIRCIMAGING.118.007753 (2018).

9. Garcia-Canadilla, P. et al. Myoarchitectural disarray of hypertrophic cardiomyopathy begins pre-birth. J Anat. 235, 962-976 (2019).

10. Mirea, I. et al. Very high-resolution imaging of post-mortem human cardiac tissue using X-ray phase contrast tomography. Functional Imaging and Modeling of the Heart. 172-179 (Springer International Publishing; 2015).

11. Varray, F. et al. Extraction of the 3D local orientation of myocytes in human cardiac tissue using X-ray phase-contrast microtomography and multi-scale analysis. Med Image Anal. 2017;38:117-132. doi: 1016/j.media.2017.02.006.

12. Kaneko, Y. et al.. Intact imaging of human heart structure using X-ray phase-contrast tomography. Pediatr Cardiol. 38, 390-393 (2017).

13. Tsukube, T. et al. Impact of synchrotron radiation-based X-ray phase-contrast tomography on understanding various cardiovascular surgical pathologies. Gen Thorac Cardiovasc Surg. 63, 590592 (2015).

14. Shinohara, G. et al. Three Dimensional Visualization of Human Cardiac Conduction Tissue in Whole Heart Specimens by High-Resolution Phase-Contrast CT Imaging Using Synchrotron Radiation. World J Pediatr Congenit Heart Surg. 7, 700-705 (2016).

15. Dejea, H. et al. Microstructural analysis of cardiac endomyocardial biopsies with synchrotron radiation-based X-Ray phase contrast imaging. Functional Imaging and Modeling of the Heart. 2331 (Springer International Publishing, 2017).

16. Baličević, V. et al. Assessment of Myofiber Orientation in High Resolution Phase-Contrast CT Images. Functional Imaging and Modeling of the Heart. 111-119 (Springer International Publishing, 2015).

17. Dube, K.N. et al. Recapitulation of developmental mechanisms to revascularize the ischemic heart. JCl Insight. 2, e96800; 10.1172/jci.insight.96800 (2017).

18. Del Monte, G., Harvey, P. An endothelial contribution to coronary vessels. 151, 932-934 (2012). 
19. Das, S. et al. A unique collateral artery development program promotes neonatal heart regeneration. 176, 1-15 (2018).

20. Konstam, M.A., Kramer, D.G., Patel, A.R., Maron, M.S., Udelson, J.E. Left ventricular remodeling in heart failure. Current Concepts in clinical significance and assessment. JACC Cardiovasc Imaging. 4, 98-108 (2011).

21. Seidel, T., Edekmann, J.C., Sachse, F.B. Analysing Remodelling of Cardiac Tissue: A comprehensive approach based on confocal microscopy and 3D reconstructions. Ann Biomed Eng. 44, 1436-1448 (2016).

22. Sands, G.B. et al. Automated imaging of extended tissue volumes using confocal microscopy. Micro Res Techn. 67, 227-239 (2005).

23. Pinali, C., Kitmitto, A. Serial block face scanning electron microscopy for the study of cardiac muscle ultrastructure at nanoscale resolutions. J Mol Cell Cardiolog. 76, 1-11 (2014).

24. Wang, J. et al. Pathological mechanism for delayed hyperenhancement of chronic scarred myocardium in contrast agent enhanced magnetic resonance imaging. PLoS One. 9, e96463; 10.1371/journal.pone.0096463 (2014).

25. Naumova, A.V., Yarnykh, V.L. Assessment of heart microstructure. From mouse to man. 129, 17201722 (2014).

26. Froeling, M., Strijkers, G.J., Nederveen, A.J., Chamuleau, S.A., Luijten, P.R. Diffusion tensor MRI of the heart- in vivo imaging of myocardial fiber architecture. Curr Cardiovasc Imaging Rep.7, 9276; 10.1007/s12410-014-9276-y (2014).

27. Wu, M.T. et al. Diffusion tensor magnetic resonance imaging mapping the fiber architecture remodelling in human myocardium after infarction: correlation with viability and wall motion. Circulation. 114, 1036-1045 (2006).

28. Geerts-Ossevoort, L., Bovendeerd, P., Prinzen, F., Arts, T., Nicolay, K. Myofiber orientation in the normal and infarcted heart, assessed with MR-diffusion tensor imaging. Comput Cardiol.28, 621-624 (2001).

29. Bernus, O. et al. Comparison of diffusion tensor imaging by cardiovascular magnetic resonance and gadolinium enhanced 3D image intensity approaches to investigation of structural anisotropy in explanted rat hearts. J Cardiovasc Magn Reson. 17, 31 (2015).

30. Hervas, A. et al. Inhomogeneity of collagen organization within fibrotic scar after myocardial infarction: results in a swine model and in human samples. J Anat.228, 47-58 (2016).

31. Whittaker, P., Boughner, D.R., Kloner, R.A. Analysis of healing after myocardial infarction using polarized light microscopy. Am J Path. 134, 879-893 (1989).

32. Goergen, C.J., Chen, H.H., Sakadžić, S., Srinivasan, V.J., Sosnovik, D.E. Microstructural characterization of myocardial infarction with optical coherence tractography and two-photon microscopy. Physiol Rep. 4, e12894; 14814/phy2.12894 (2016).

33. Zimmerman, S.D., Karlon, W.J., Holmes, J.W., Omens, J.H., Covell, J.W. Structural and mechanical factors influencing infarct scar collagen organization. Am J Physiol Heart Circ Physiol.278, H194H200 (2000). 
34. Vila-Comamala, J. et al. Development of Laboratory Grating-based X-ray Phase Contrast Microtomography for Improved Pathology. Microsc Microanal. 24,192-193 (2018).

35. Viermetz, M.P. et al. High resolution laboratory grating-based x-ray phase-contrast CT. Sci Rep. $\mathbf{8}$, 15884; 10.1038/s41598-018-33997-5 (2018).

36. Otendal, M., Tuohima, A., Vogt, U., Hertz, H.M.. A 9 keV electron-impact liquid-gallium-jet x-ray source. Rev Sci Instrum. 79, 016102; 10.1063/1.2833838 (2008).

37. Reichardt, M., Topperwien, M., Khan, A., Alves, F., Salditt, T. Fiber orientation in a whole mouse heart reconstructed by laboratory phase-contrast micro-CT. J Med Imag. 7, 023501; 10.1117/1.JMI.7.2.023501 (2020).

38. Nakajima, K. Compact X-ray sources: Towards a table-top free-electron laser. Nat Phys. 4, 92-93 (2008).

39. Schlenvoigt, H.P. et al. A compact synchrotron radiation source driven by a laser-plasma wakefield accelerator. Nat Phys. 4, 130-133 (2007).

40. Eggl, E. et al. X-ray phase-contrast tomography with a compact laser-driven synchrotron source. Proc Natl Acad Sci. 112, 5567-5572 (2015).

41. Cardin, S. et al. Role for MicroRNA-21 in atrial profibrillatory fibrotic remodeling associated with experimental post-infarction heart failure. Circ Arrhythm Electrophysiol. 5, 1027-1035 (2012).

42. Marone, F., Stampanoni, M. Regridding reconstruction algorithm for real-time tomographic imaging. $J$ Synchrotron Radiat.19, 1029-37 (2012).

43. Paganin, D., Mayo, S.C., Gureyev, T.E., Miller, P.R., Wilkins, S.W. Simultaneous phase and amplitude extraction from a single defocused image of a homogeneous object. J Microsc.206, 33-40 (2002).

44. Schindelin, J. et al. Fiji: an open-source platform for biological-image analysis. Nat methods.9, 676682 (2012).

45. Schindelin, J., Rueden, C.T., Hiner, M.C., Eliceiri, K.W. The ImageJ ecosystem: an open platform for biomedical image analysis. Mol Reprod Dev.82, 518-529 (2015).

46. Berg, S. et al. llastik: interactive machine learning for (bio)image analysis. Nat Methods. 16, 12261232 (2019).

47. Captur, G. et al. The embryological basis of subclinical hypertrophic cardiomyopathy. Sci Rep. 6, 27714; 10.1038/srep27714 (2016).

48. Kikinis, R., Pieper, S.D., Vosburgh, K. 3D Slicer: a platform for subject-specific image analysis, visualization, and clinical support in Intraoperative imaging and image-guided therapy (ed Jolesz, F.A.) 277-289 (Springer, 2014).

49. Fedorov, A. et al. 3D Slicer as an image computing platform for the quantitative imaging network. Magn Reson Imaging. 30, 1323-1341 (2012).

50. Centre for Integrative Biomedical Computing at the University of Utah Scientific Computing and Imaging Institute. Seg3D: Volumetric Image Segmentation and Visualization. 2016. v2.4.4. Available from: http://www.seg3d.org 


\section{Figures}

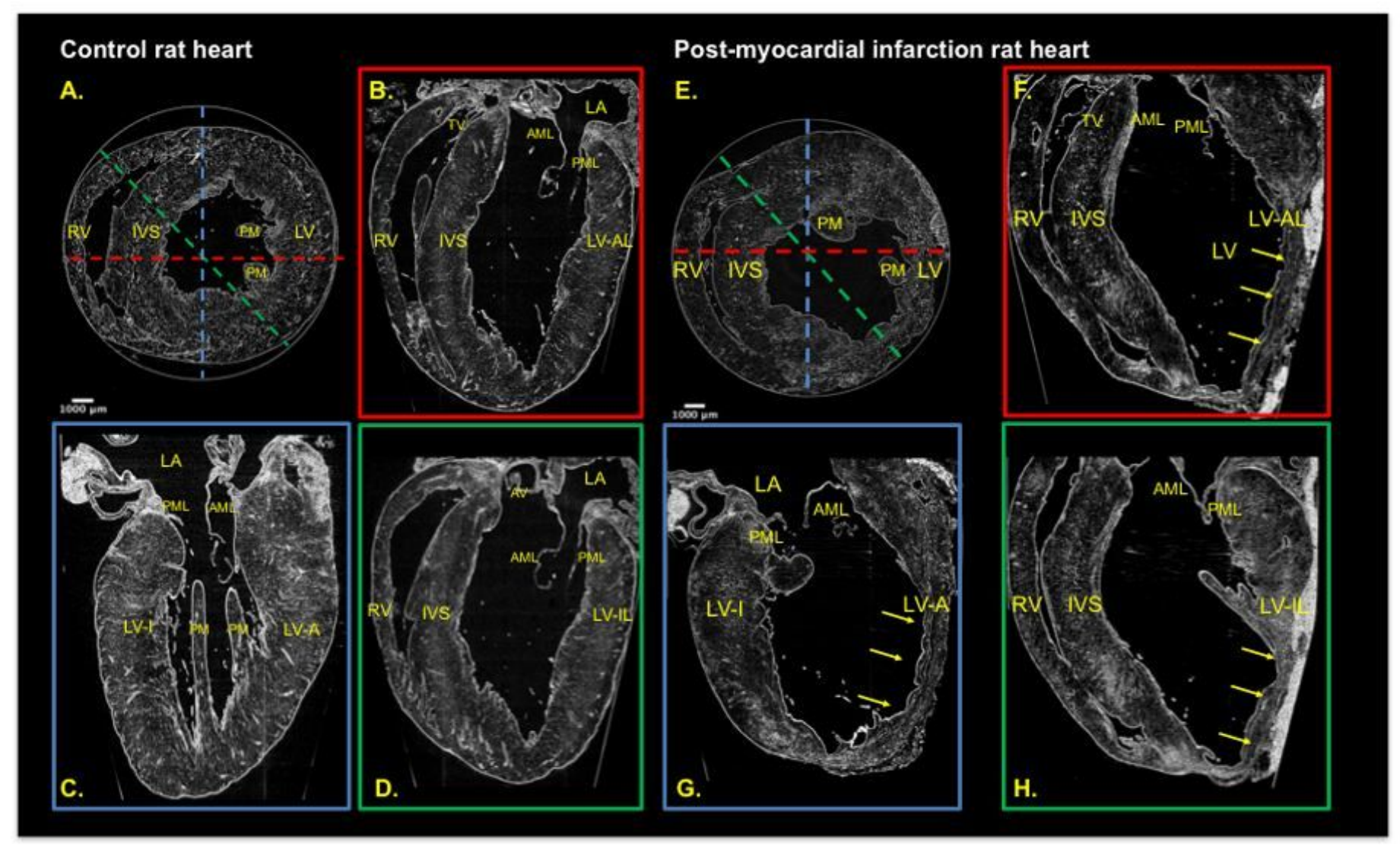

Figure 1

PB X-PCl images reconstructed as conventional imaging views. Control heart from one healthy rat was used for left panel images, and infarcted heart from rat with induced myocardial infarction was used for the right panel. Left panel - control; right panel - infarct. A. Short axis view, B. Apical 4 chamber view, C. Apical 2 chamber view, D. Apical 5 chamber view; E. Short axis view, F. Apical 4 chamber view, G. Apical 2 chamber view, $\mathrm{H}$. Apical 5 chamber view. The dashed lines indicate the position of the slice perpendicular to the short axis, producing a specific longitudinal view. Short yellow arrows indicate the infarct areas with clear aneurysm formation. LV: left ventricle, RV: right ventricle, PM: papillary muscle, IVS: interventricular septum, PML: posterior leaflet of mitral valve, AML: anterior leaflet of mitral valve, TV: tricuspid valve, LA: left atrium, AV: aortic valve, LV-AL: anterolateral wall of the left ventricle, LV-A: anterior wall of the left ventricle, LV-I: inferior wall of the left ventricle, LV-IL: inferolateral wall of the left ventricle. 


\section{Wall thickness}
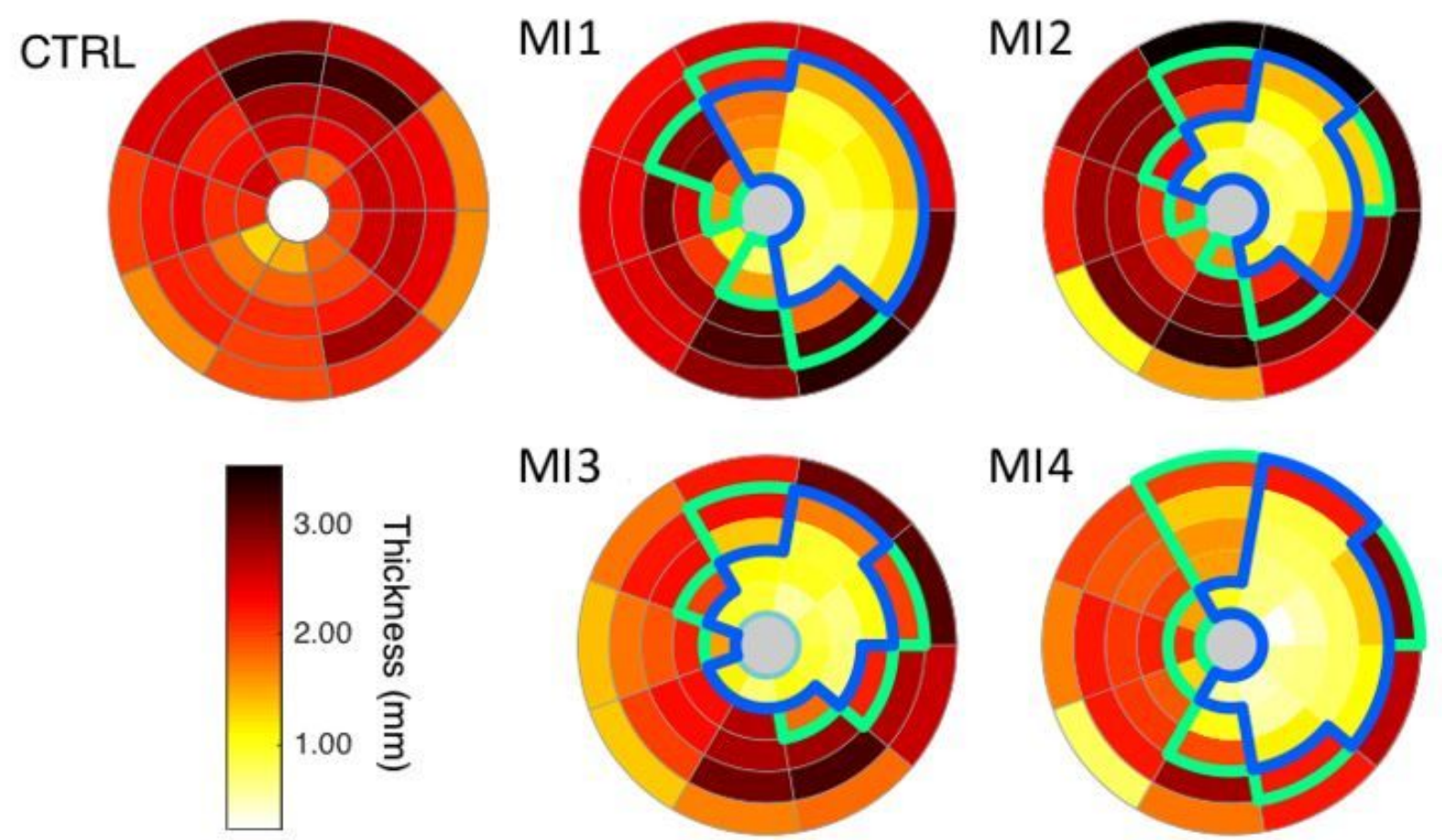

\section{Figure 2}

Schematic representation of the 45-segment model visualizing regional wall thickness of LV rat hearts affected by a myocardial infarction as compared to the control. For this figure, 5 rat hearts were used (control heart from the healthy rat, and 4 infarcted hearts from 4 rats with induced myocardial infarction). The segments corresponding to $\mathrm{Ml}$ (blue), and peri-MI areas (green) are highlighted. CTRL: control rat heart; MI 1-4: post-MI rat hearts 


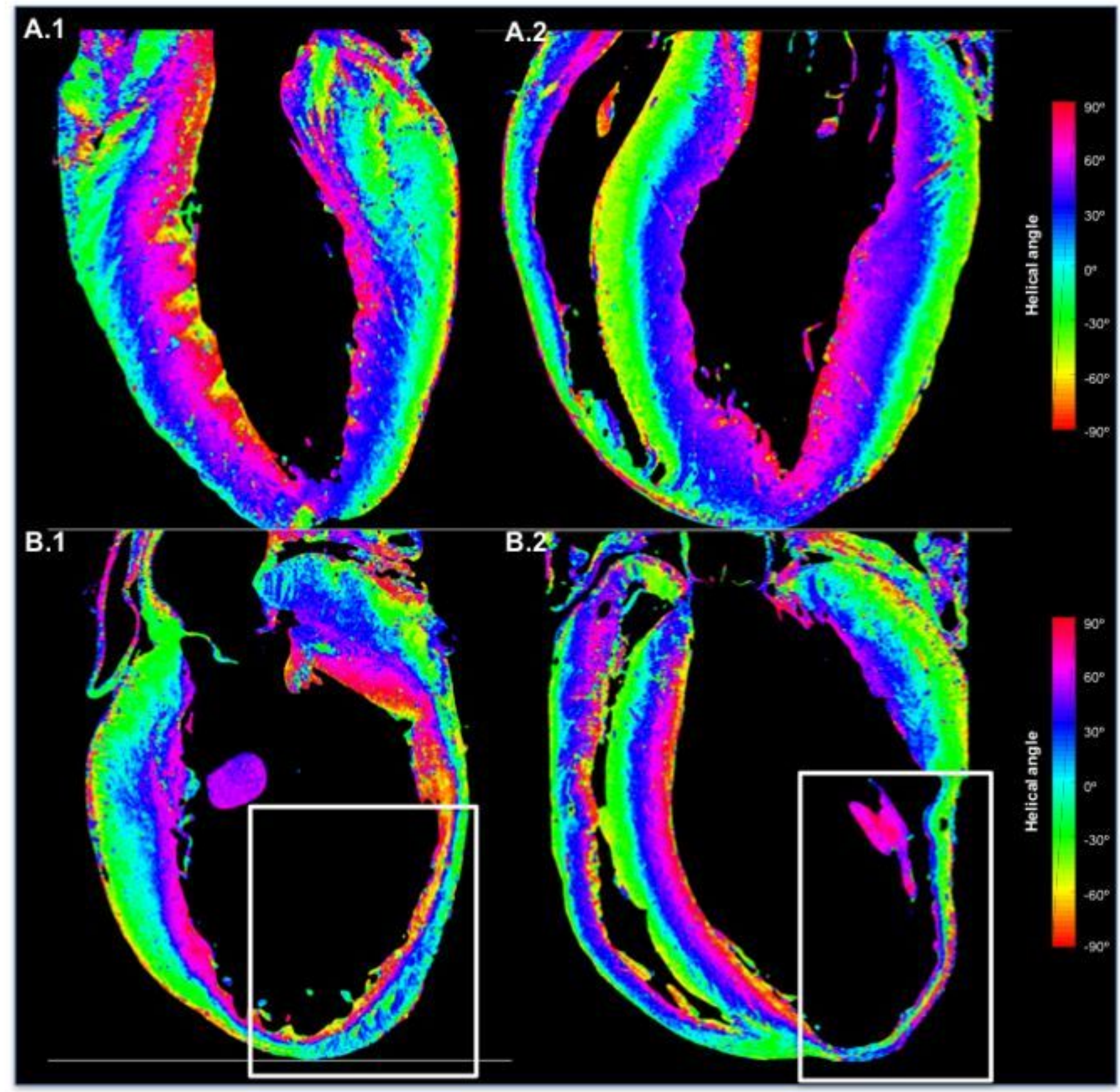

Figure 3

Longitudinal visualization of the local helical angles (HA) of myocyte aggregates. One control heart from a healthy rat, and one infarcted heart from a rat with induced myocardial infarction was used. Different colors represent different HAs. A.1: Apical 2 chamber view, and A.2: Apical 4 chamber view of the control heart. B.1: Apical 2 chamber view of the infarcted heart showing a large aneurysm of the anterior wall (white rectangle). B.2: Apical 4 chamber view of the same infarcted heart showing the apical extend of the aneurism (white rectangle). 


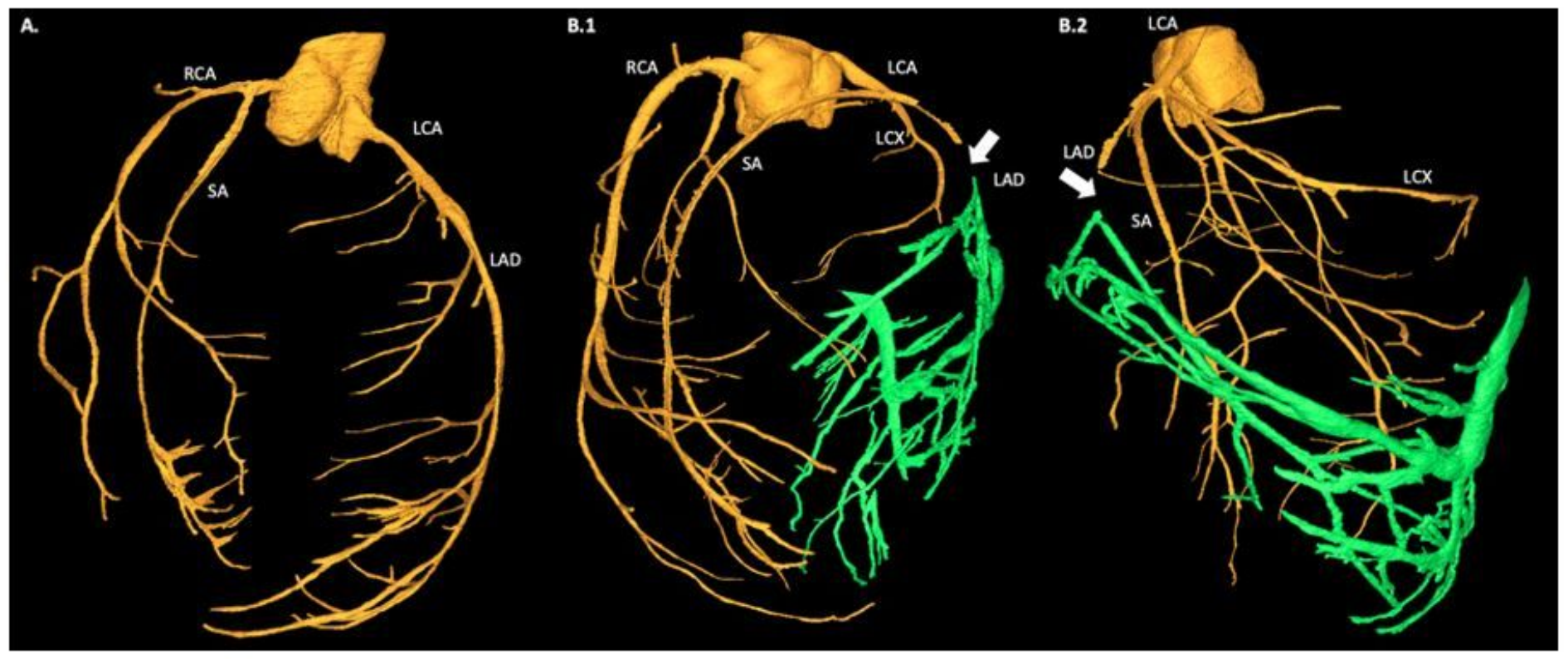

Figure 4

3D Rendering of the segmented vascular tree. For this rendering one control heart from a healthy rat, and one infarcted heart from a rat with induced myocardial infarction was used. A. Control rat heart. B.1 and B.2 Post-myocardial infarction heart. Coronary arteries branching is represented in yellow. Green color indicates increased vascular spaces in the infarct area. White arrow indicates site of left coronary artery ligation. RCA: right coronary artery, LCA: left coronary artery, SA: septal artery, LCX: left circumflex artery, LAD: left anterior descending artery 


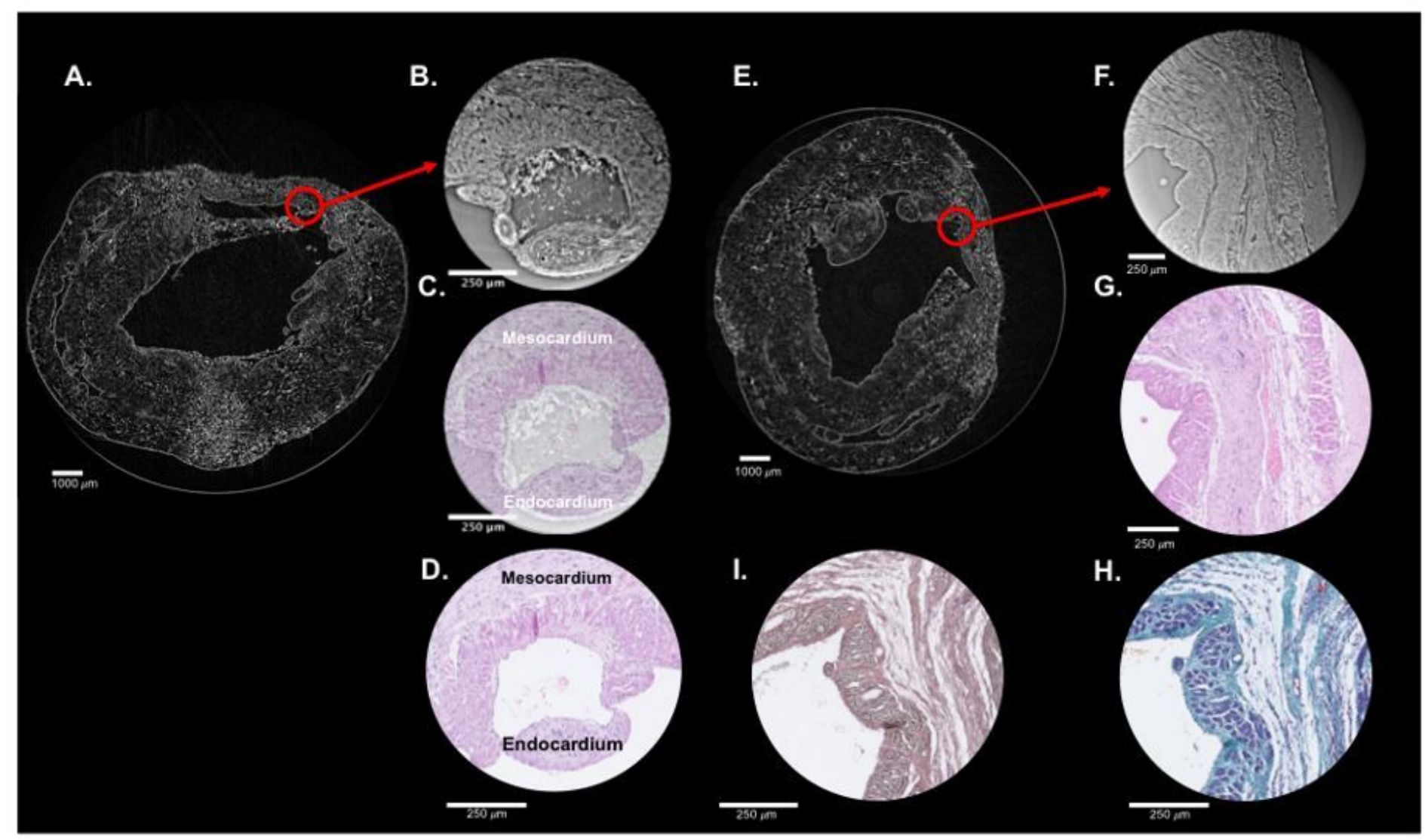

\section{Figure 5}

Comparing PB-XPCl virtual histology and conventional light microscopy. For this image one infarcted heart from a rat with induced myocardial infarction was used. Left panel- direct comparison of the identical myocardial area imaged by PB X-PCl virtual histology and light microscopy. A. Low resolution $\mathrm{PB} X-\mathrm{PCl}$ of the infarcted heart. Red circle indicates part of the infarcted area that was imaged by high resolution PB X-PCI. B. High resolution PB X-PCI image of the selected area. C. Overlap of the same slice by $\mathrm{PB}$ X-PCl and light microscopy. D. Hematoxylin and eosin-stained light microscopy (magnification 100x). On every image from B. to D., a varying orientation of cardiomyocytes aggregates may be observed- at endocardial side longitudinally oriented; while towards the mesocardium are circumferentially oriented. Right panel- E. PB X-PCI image at low resolution representing the short axis view of the left ventricle and indicating the region of interest used for acquisition of the high-resolution PB X-PCl showed on F. G. Hematoxylin and eosin staining of the identical region of the same heart showing high resemblance to PB X-PCl virtual histology image. H. Mallory's trichrome staining showing increased amounts of mature collagen (shown in blue color) in the area of myocardial infarction, i.e. scar tissue. I. Gömöri's silver impregnation staining showing no reticular fibers in the region of interest (no areas stained in black on the sample). 
A.1

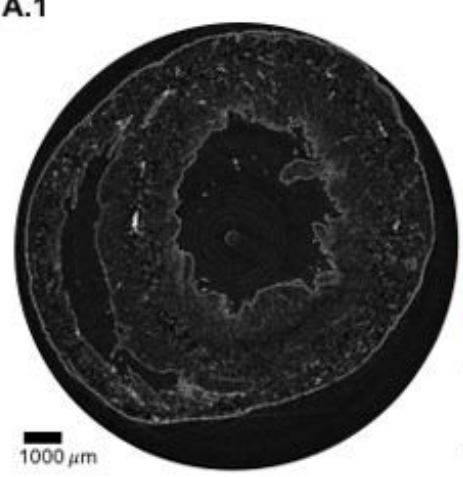

A.3

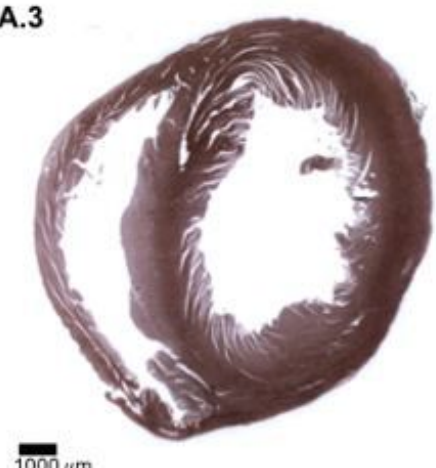

A.2

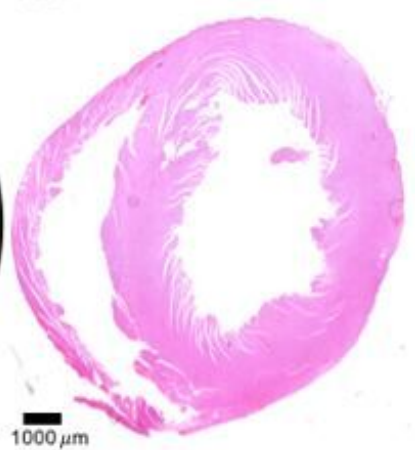

A.4

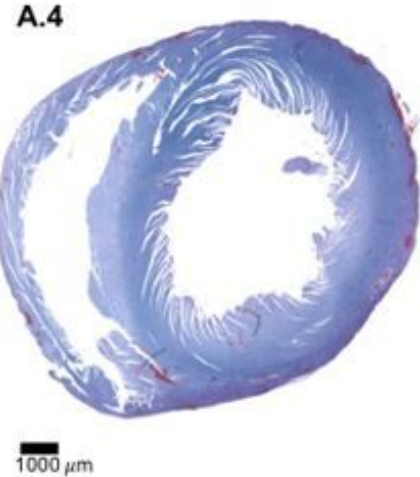

B.1

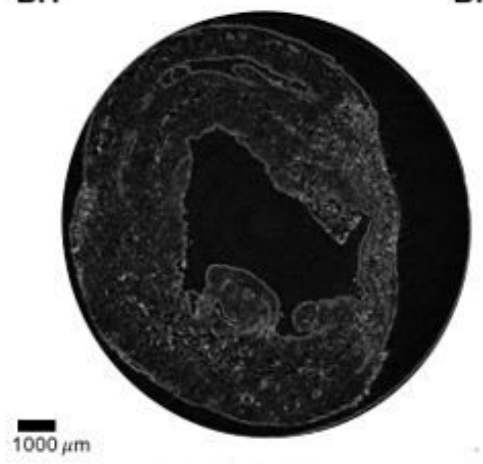

B.2

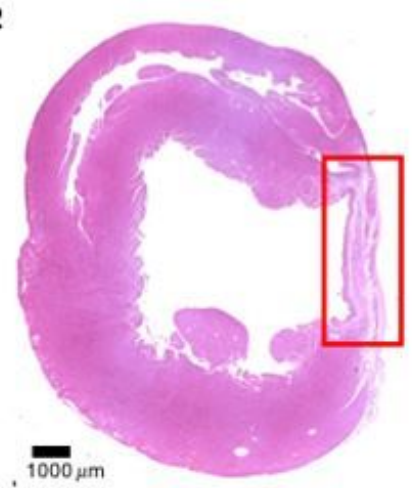

B.3

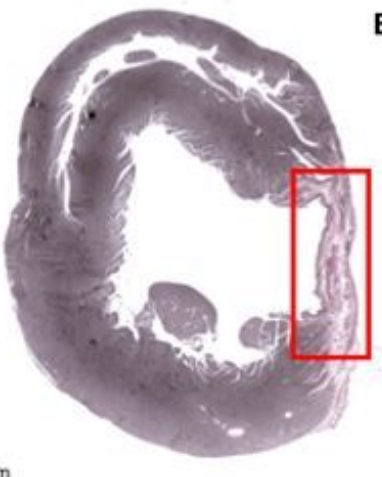

B.4

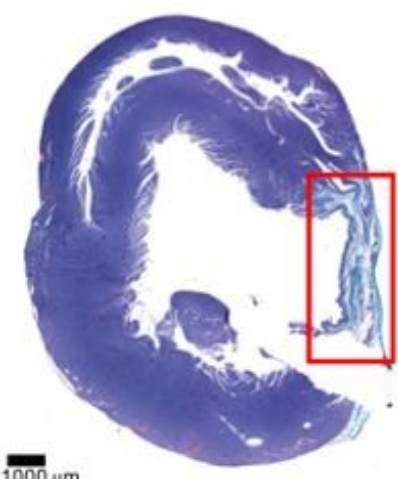

Figure 6

Comparison of PB X-PCl virtual histology and classical histology. One control heart from a healthy rat, and one infarcted heart from a rat with induced myocardial infarction was used. A.1-A.4 Control rat heart in short axis view. A.1 PB X-PCI virtual histology image. A.2 Hematoxylin-eosin, A.3 Gömöri's silver staining, A.4 Mallory's trichrome of the same heart. B.1-B.4 Myocardial infarction rat heart. B.1 PB X-PCI virtual histology image. B.2 Hematoxylin-eosin, B.3 Gömöri's silver staining, B.4 Mallory's trichrome of the same heart. Red boxes on B images indicate area of scar following myocardial infarction.

\section{Supplementary Files}

This is a list of supplementary files associated with this preprint. Click to download.

- Videol.mov

- Videoll.mov

- Supplementarymaterial.docx

- Videolll.mov 